\title{
Clinical and immunological characteristics of Polish patients with systemic lupus erythematosus
}

\author{
Martyna Tomczyk-Socha a, B-E, Hanna Sikorska-Szaflik2, B-E , Marek Frankowski, B-D, \\ Karolina Andrzejewska ${ }^{3, B-D}$, Agnieszka Odziomek ${ }^{4, B-D}$, Magdalena Szmyrka ${ }^{3, A, B, E, F}$ \\ ${ }^{1}$ Department and Clinic of Ophthalmology, Wroclaw Medical University, Poland \\ ${ }^{2} 11^{\text {st }}$ Department and Clinic of Pediatrics, Allergology and Cardiology, Wroclaw Medical University, Poland \\ ${ }^{3}$ Department and Clinic of Rheumatology and Internal Medicine, Wroclaw Medical University, Poland \\ ${ }^{4}$ Department of Dermatology, Regional Specialized Hospital, Wrocław, Poland \\ A - research concept and design; B - collection and/or assembly of data; C - data analysis and interpretation; \\ $D$ - writing the article; $E$ - critical revision of the article; $F$ - final approval of the article
}

\section{Address for correspondence}

Martyna Tomczyk-Socha

E-mail:martyna_tomczyk@wp.pl

Funding sources

None declared

Conflict of interest

None declared

Received on February 2, 2016

Reviewed on August 26, 2016

Accepted on 0ctober 17, 2016

DOI

10.17219/acem/65860

\section{Copyright}

Copyright by Author(s)

This is an article distributed under the terms of the

Creative Commons Attribution Non-Commercial License

(http://creativecommons.org/licenses/by-nc-nd/4.0/)

\section{Abstract}

Background. Systemic lupus erythematosus (SLE) is a chronic autoimmune disease with varied clinical manifestations, which creates difficulties and delays in establishing a diagnosis.

Objectives. The aim of this study was to evaluate the prevalence and nature of the clinical symptoms of SLE, both at the onset of the disease and in its further course. An attempt to assess the immunological characteristics of the patients and to analyze autoantibodies variability over time was also made.

Material and methods. This retrospective study included 71 Caucasian patients, 63 women and 8 men, meeting the criteria for diagnosis of SLE according to ACR.

Results. The ratio of women to men was approximately 7.9:1. The average age of the onset of SLE was 31.5 years. The average time from the onset of symptoms to diagnosis was 5 years. The most common first manifestation of SLE were joint and muscles symptoms $-71.8 \%$, skin lesions $-69.0 \%$, fever $-57.7 \%$. The main symptoms in the further course of the disease were neurological disorders $-69.0 \%$, joint and muscle changes $-67.7 \%$, and general symptoms $-59.2 \%$. There was an increase in the incidence of renal involvement and neurological symptoms throughout the disease course. The most commonly detected antibodies were anti-dsDNA - 47.9\%, anti-Ro/SSA - 40.8\%, anti-nucleosomal antibodies - 29.6\%, and lupus anticoagulant $-22.5 \%$. A panel of antibodies typically did not change.

Conclusions. There is no typical clinical picture of SLE, the population suffering from this disease is very various. Therefore, early and accurate diagnosis can be a big challenge for any clinician, which justifies the need for this type of study to better characterize the disease.

Key words: systemic lupus erythematosus, SLE, course of SLE, onset of SLE 


\section{Introduction}

Systemic lupus erythematosus (SLE) is a chronic inflammatory autoimmune disease. It involves many organs and systems, mainly the skin, joints, kidneys, and the central nervous system. ${ }^{1}$ In the course of the disease, general symptoms such as fever, weight loss, and fatigue are commonly seen. The first manifestations of SLE most often occur in young adults. ${ }^{2}$

To establish the diagnosis of SLE, patients have to fulfill at least 4 classification criteria developed by the American College of Rheumatology (ACR). ${ }^{3,4}$ Based on the binomial coefficient, there are 330 combinations of symptoms that can be used to determine the diagnosis of SLE.

Due to this heterogeneous picture of the disease, SLE diagnosis is often delayed in relation to the appearance of the first symptoms. The occurrence of periods of exacerbation and remission is characteristic, and the course of the disease may take different forms, from mild to severe and even to life threatening., 5

The aim of this study was to assess the incidence, clinical symptoms, dominant signs which indicate SLE, and the dominant signs in the course of the disease. Particular attention was paid to the delay in establishing the diagnosis of SLE in relation to the onset of symptoms, as well as to the average age of onset.

An attempt to assess the immunological characteristics of the patients was also made. Both the incidence of various autoantibodies at the time of establishing diagnosis and their variability over time were tested. The association between the presence of specific antibodies and the involvement of various systems, as well as the severity of the disease were examined.

\section{Material and methods}

The study records of 71 Caucasian patients, including 63 women (89\%) and 8 men (11\%) admitted to the Department of Rheumatology and Internal Medicine of the University Hospital in Wrocław in 2009-2011, were reviewed and included in a retrospective analysis. All patients fulfilled the criteria for the classification of SLE given by the ACR. Data was obtained from the patients' records and questionnaires on the basis of clinical symptoms and laboratory results. General symptoms (fever - more than $38^{\circ} \mathrm{C}$ after the exclusion of infection, weight loss, fatigue), skin lesions, mucosal changes, joint and muscle ailments, sensitivity to the sunlight, hair loss, lymphadenopathy, inflammation of the serous membranes, renal involvement (proteinuria, hematuria, pyuria), neuropsychiatric disorders (e.g., headaches, mood disorders, cognitive disorders), hematological symptoms (anemia, leukopenia, thrombocytopenia), sicca syndrome, and antiphospholipid syndrome were taken into account.

The presence of antinuclear antibodies, antiphospholipid antibodies, lupus anticoagulant was recorded. Antinuclear
(ANA) and anti-double stranded DNA (anti-dsDNA) antibodies were routinely detected by indirect immunofluorescence on HEp-2 cells and Crithidia luciliae substrate, respectively. Antiextractable nuclear antigens (anti-Ro/ SSA, anti-La/SSB, anti-Sm, and anti-RNP) antibodies were detected by qualitative enzyme-linked immunosorbent assays (ELISA), while the lupus anticoagulant (LA) was detected according to the guidelines of the International Society of Thrombosis and Hemostasis.

Specific symptoms and antibodies present in patients at the moment of diagnosis were monitored and compared at a later time in the course of the disease. The dominant frequency of symptoms and their correlation with the results of immunological studies were also considered.

The data is presented as mean values, standard deviations and percentages.

\section{Results}

In this study, the ratio of women to men was approximately 7.9:1, which correlates with the European SLE population. ${ }^{5}$ The average age when the first symptoms appeared was 31.5 years (SD 11.8). The percentage of people whose onset began before the age of 14 years was $6 \%$, and those with the first symptoms over the age of 50 years was $8 \%$. The mean age of the establishing the diagnosis of SLE was 36.5 years (SD 13.94); therefore, the delay in diagnosis was 5 years (SD 5.24).

In the study group, joint and muscle symptoms dominated at diagnosis. They were present in 51 of 71 patients, which constituted $71.8 \%$. The second most common signs were skin lesions $-69.0 \%$. General symptoms were present in $57.7 \%$ of patients, including the dominant fever. Photosensitivity was observed in $52.1 \%$ of patients.

The average time between the appearance of the first symptoms and the conducted study examination was about 9 years, which allowed us to assess the subsequent course of the disease, in which neurological and psychiatric symptoms were dominant (occurring in 69.0\%). The most frequent were headaches, mood disorders (depression), cognitive disorders, and cerebrovascular disease. Changes in joints and muscles were also important, as reported by $67.7 \%$ of the study group. General symptoms were present in $59.2 \%$ of patients, and the most commonly observed symptom was fatigue.

The prevalence of all symptoms evaluated in this study, both at the beginning and in the later stages of the disease, are included in Table 1 and Fig. 1 (A, B).

\section{General symptoms}

At least 1 of the general symptoms occurred in $62.0 \%$ of people in the early stage of disease and in $59.2 \%$ in the later course of SLE. Initially, the dominant general symptom was fever, which was observed in $47.9 \%$ 
of patients. Fatigue (36.6\%) and weight loss (26.8\%) occurred less often. In the later stages of the disease, fever occurred less frequently (38.0\%), and the most common symptom was fatigue (47.9\%) and weight loss (19.7\%).

\section{Musculocutaneous symptoms}

In our sample, the incidence of skin lesions at the disease onset was $69.0 \%$, decreasing thereafter to $47.9 \%$. Hypersensitivity to sunlight was observed in $52.1 \%$ of patients at the onset of the disease, and in $28.2 \%$ in its later course.

Table 1. Prevalence of all symptoms evaluated in this study, both at the beginning and in the later stages of SLE

\begin{tabular}{|c|c|c|c|c|}
\hline \multicolumn{5}{|c|}{ The incidence of selected symptoms } \\
\hline \multirow{2}{*}{ symptom } & \multicolumn{2}{|c|}{$\begin{array}{l}\text { the initial period } \\
\text { of the disease }\end{array}$} & \multicolumn{2}{|c|}{$\begin{array}{l}\text { the later period } \\
\text { of the disease }\end{array}$} \\
\hline & $\begin{array}{c}\text { number } \\
\text { of patients }\end{array}$ & $\begin{array}{c}\% \\
(\mathrm{~N}=71)\end{array}$ & $\begin{array}{l}\text { number } \\
\text { of patients }\end{array}$ & $\begin{array}{c}\% \\
(\mathrm{~N}=71)\end{array}$ \\
\hline Joint and muscle changes & 51 & 71.8 & 48 & 67.6 \\
\hline Skin changes & 49 & 69.0 & 34 & 47.9 \\
\hline Neuropsychiatric symptoms & 42 & 59.2 & 51 & 71.8 \\
\hline Sunlight sensitivity & 37 & 52.1 & 20 & 28.2 \\
\hline Fever & 34 & 47.9 & 27 & 38.0 \\
\hline Fatigue & 26 & 36.6 & 34 & 47.9 \\
\hline Anemia & 24 & 33.8 & 30 & 42.3 \\
\hline Leucopenia & 23 & 32.4 & 24 & 33.8 \\
\hline Alopecia & 21 & 29.6 & 20 & 28.2 \\
\hline Weight loss & 19 & 26.8 & 14 & 19.7 \\
\hline Headache & 17 & 23.9 & 22 & 31.0 \\
\hline Proteinuria & 16 & 22.5 & 32 & 45.1 \\
\hline Serositis & 16 & 22.5 & 15 & 21.1 \\
\hline Sicca syndrome & 14 & 19.7 & 15 & 21.1 \\
\hline Thrombocytopenia & 13 & 18.3 & 9 & 12.7 \\
\hline Lymphadenopathy & 11 & 15.5 & 6 & 8.5 \\
\hline Antiphospholipid syndrome & 9 & 12.7 & 7 & 9.9 \\
\hline Hematuria & 7 & 9.9 & 18 & 25.4 \\
\hline
\end{tabular}

The presence of alopecia during the study was also pointed out, which was reported in $29.6 \%$ of patients at the time of diagnosis, dropping slightly to $28.2 \%$ as the disease progressed.

Erosions in the mouth were mainly manifested from the mucosal changes. Such changes were reported in $22.5 \%$ of patients at the time of appearance of the first symptoms, and in $19.7 \%$ at the later stages of the disease.

\section{Symptoms of joints and muscles}

The high prevalence of these symptoms was observed. About $71.8 \%$ of patients reported noncharacteristic arthritis at the beginning of the disease, and $67.7 \%$ at a later stage, which makes it the most common first symptom and the second common ailment at the course of the disease.

\section{Renal involvement}

Proteinuria, hematuria and pyuria were the clinical symptoms evaluated in the study. In the initial stage of disease, at least 1 of these symptoms occurred in $23.9 \%$ of patients. Proteinuria occurred in nearly all patients (in $22.5 \%$ of patients and in $94.1 \%$ of patients with symptoms of renal involvement), whereas hematuria and pyuria occurred less often (in $9.9 \%$ and $5.6 \%$ of all patients, respectively). In the later period, symptoms of kidney disease were reported in $52.1 \%$ of patients. The incidence of proteinuria (45.1\%) and hematuria (25.4\%) was doubled. The incidence of pyuria (15.5\%) was tripled.

\section{Neurological and psychiatric symptoms}

In the initial stage of the disease, neurological symptoms were found in $40.8 \%$ of patients. At a later course of the disease, $69.0 \%$ of the patients
A

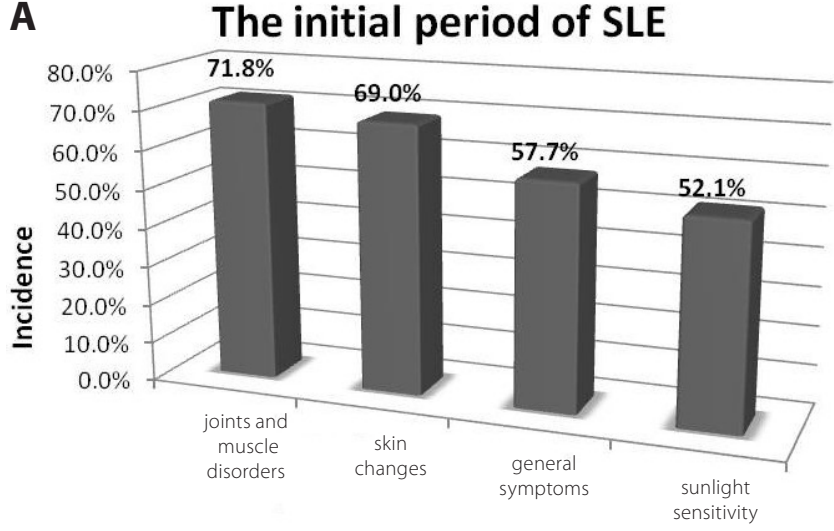

B

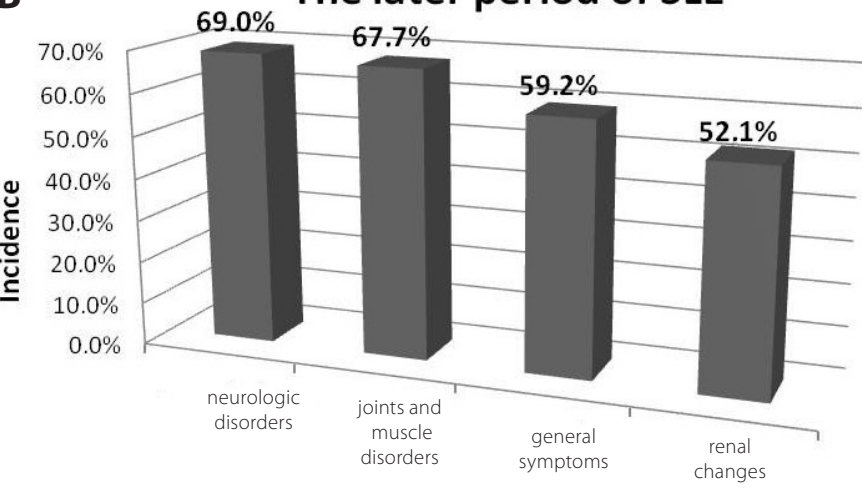


complained of at least 1 of the disorders of the nervous system. The most commonly reported disorders were headache $(23.9 \%$ at the beginning and $31.0 \%$ in the advanced stage), and affective disorders (9.9\% at diagnosis and $28.2 \%$ in the later course of the disease). Cognitive impairment in an advanced stage of SLE was manifested in $16.9 \%$ of patients. Symptoms of cerebrovascular disease, including stroke, were experienced by $9.9 \%$ of patients.

\section{Hematological changes}

In more than half of the patients (53.5\%), hematological changes (anemia, leucopenia, thrombocytopenia) were observed at the beginning of the disease. The percentage of patients who suffer from these symptoms remained the same in the later course of SLE, but with an increased number of patients who experienced at least 2 of these 3 symptoms. Leucopenia (32.4\%) and anemia (33.8\%) occurred initially with similar frequency, while in the course of the disease anemia (42.3\%) was significantly more frequently demonstrated than leucopenia (33.8\%). Thrombocytopenia was initially diagnosed in $18.3 \%$ of patients, and in the further course of SLE incidence fell about $30 \%$ and amounted to $12.7 \%$.

\section{Sjörgen's syndrome and inflammation of the serous membranes}

In the study, these symptoms were present in $22.5 \%$ of patients at the time of diagnosis. A similar incidence of occurrence $(21.1 \%)$ was noted in the further course of the disease. Sicca syndrome accompanying SLE was reported in $19.7 \%$ of patients initially and in a similar number of patients $(22.5 \%)$ in the further course of the disease.

\section{Immunological profile of patients}

ANA positive result was observed in the initial period in $87 \%$ of patients, and later in $94 \%$ of them. The full panel of antibodies was not determined for each patient in the initial stage of the disease. However, the available data shows that the most common antibodies in SLE patients in this period were anti-double stranded DNA (anti-dsDNA) and anti-Sjögren's-syndrome-related antigen A (anti-SSA autoantibodies). Similarly, in the later stages of the disease, anti-dsDNA (47.9\% of patients) and anti-SSA (40.8\%) were most common, followed by antinucleosomal antibodies (29.6\%), and lupus anticoagulant (22.5\%). Anti-ribosomal P protein was checked in 39 patients. All patients with a positive test result (6/39, $15.4 \%$ ) had neurological symptoms. However, $21.2 \%$ of patients with negative test result also had neurological symptoms.

In 40 patients, data was available from both the onset of the disease as well as during its duration; an antibody panel in these patients mostly did not change.

\section{Discussion}

Numerous studies showing the incidence of SLE in the world has been previously established. Petri et al. and Alarcon et al. studied the American population, Wang et al. investigated the Asian population, and Cervera et al. covered European population in a large cohort study Eurolupus. ${ }^{5,7-9}$ Clinical manifestation of SLE varies considerably depending on the geographical region and, therefore, it was decided that this study should be performed based on the Polish population. Data was collected in 1 region of Poland, the Lower Silesia.

The presented results are similar to those shown in the study of the Euro-Lupus. Despite the significant difference in the number of patients in groups, similar basic symptoms of SLE were observed in both cases. These include changes in joints and muscles, as well as skin.

However, a considerably lower number of documented changes in kidney disease symptoms, such as proteinuria, hematuria and pyuria were found at the beginning of the disease. Nevertheless, analyzing the incidence of nephropathy in the further course of SLE, its presence was observed in $50 \%$ of patients (Euro-Lupus 27.9\%). Similar results were included in the research on the US population (55.6\%). In the Asian population, nephropathy was present in $74 \%$ of patients. This shows that the variability in the incidence of particular symptoms differs significantly depending on the geographical region and needs to be studied in particular groups to determine the natural history of SLE.

Clinical manifestation of symptoms also varies with the duration of illness. Initial dominance of presentation of skin symptoms is later replaced by symptoms of the nervous system, such as headaches, mood disorders, cognitive disorders, and vascular changes. The incidence of depression drastically increased. This indicates the need for more frequent neurological, psychological and mental health consultations throughout the course of the disease.

SLE is a disease appearing mainly in young adults. In the present study, the vast majority (86\%) of the first symptoms of SLE appeared at the age of 15-49 years, but $6 \%$ were observed at a younger age, and $8 \%$ in the elderly. In the group of $50+$ years of age, female dominance is not as strongly pronounced (only 67\%), while in the youngest group it is equal to $100 \%$ (in the whole study population $89 \%)$. In neither the younger nor the older age group was there a significant difference in the incidence of individual symptoms of SLE in comparison to the general population, as suggested by other studies. ${ }^{5,10-13}$

The observed time from the appearance of the first symptoms of the disease to the time when 4 of the ACR criteria were met was 5 years, and it is significantly longer compared to a European study (2 years). ${ }^{5}$ This difference may be due to the milder initial course of the disease in the Polish population, or to other methods of collecting information (Euro-Lupus is a prospective study; the current study collected data retrospectively). 
Although headaches are the most frequently reported neuropsychiatric symptoms, they have a low specificity for SLE and are classified as minor syndromes. Studies also demonstrated that headaches are not more common in people suffering from lupus compared to the population of healthy people, and characteristic features of the pain were not observed. ${ }^{14,15}$

Depression may also result from causes not directly related to the SLE disease process. It can be due to steroid therapy, among others, and likely depends on the predisposition of the individual. The appearance of seizures and vascular lesions in the brain (stroke, transient ischemic attack - TIA) are related largely to the presence of antiphospholipid antibodies, which are responsible for the occurrence of prothrombotic state. ${ }^{16,17}$

In the study population, the most common symptoms at presentation were changes in muscle and joint system, skin, general symptoms, and sensitivity to UV light. As the disease progresses, the percentage incidence of neurological symptoms and renal involvement are increased, while syndromes from joint and muscle system and the skin continue to be an important concern. The average time from onset of symptoms to diagnosis was 5 years. Profile of autoantibodies did not change during the course of the disease with anti-dsDNA and anti-SSA as the most common autoantibodies.

SLE as a systemic disease is characterized by the presence of many symptoms associated with the activity of various organs. Most of them are non-specific, and even those characteristics of lupus are present only in some patients. There is no typical clinical picture of SLE; therefore, the patient population suffering from this disease is very various. Differences in the course of the disease are also evident due to the geographic area. Therefore, early diagnosis can be a big challenge for any clinician, which justifies the need for this type of study to better characterize the disease.

\section{References}

1. Williams AE, Crofts G, Teh LS. "Focus on feet" - The effects of systemic lupus erythematosus: A narrative review of the literature. Lupus. 2013;22(10):1017-1023.

2. Ahmadpoor P, Dalili N, Rostami M. An update on pathogenesis of systemic lupus erythematosus. Iran J Kidney Dis. 2014;8(3):171-184.

3. Tan EM, Cohen AS, Fries JF, et al. The 1982 revised criteria for the classification of systemic lupus erythematosus. Arthritis Rheum. 1982;25:1271-1277.

4. Hochberg MC. Updating the American College of Rheumatology revised criteria for the classification of systemic lupus erythematosus. Arthritis Rheum. 1997;40(9):1725.

5. Cervera R, Khamashta MA, Hughes GRV. The Euro-Lupus project: Epidemiology of systemic lupus erythematosus in Europe. Lupus. 2009;18:869-874.

6. Majdan M. Toczeń rumieniowaty układowy. Reumatologia. 2012;50(2):103-110.

7. Petri M. The effect of race on the presentation and course of SLE in the United States. Arthritis Rheum. 1997;40(Suppl):162.

8. Alarcón GS, McGwin Jr G, Petri M, Reveille JD, Ramsey-Goldman R, Kimberly RP. Baseline characteristics of a multiethnic lupus cohort: PROFILE. Lupus. 2002;11:95-101.
9. Wang F, Wang CL, Tan CT, Manivasagar M. Systemic lupus erythematosus in Malaysia: A study of 539 patients and comparison of prevalence and disease expression in different racial and gender groups. Lupus. 1997;6:248-253.

10. Brunner HI, Gladman DD, Ibañez D, Urowitz MD, Silverman ED. Difference in disease features between childhood-onset and adult-onset systemic lupus erythematosus. Arthritis Rheum. 2008;58:556-562.

11. Dung NT, Loan HT, Nielsen S, Zak M, Petersen FK. Juvenile systemic lupus erythematosus onset patterns in Vietnamese children: A descriptive study of 45 children. Pediatric Rheumatology Online J. 2012;10:38.

12. Costallat LT, Coimbra AM. Systemic lupus erythematosus: Clinical and laboratory aspects related to age at disease onset. Clin Exp Rheumatol. 1994;12(6):603-607.

13. Tomic-Lucic A, Petrovic R, Radak-Perovic M, et al. Late-onset systemic lupus erythematosus: Clinical features, course, and prognosis. Clin Rheumatol. 2013;32(7):1053-1058.

14. Hajduk A, Smoleńska Ż, Nowicka-Sauer K, et al. Neuropsychiatric syndromes in systemic lupus erythematosus patients. Reumatologia. 2012;50(6):493-500.

15. Ainiala H, Loukkola J, Peltola J, Korpela M, Hietaharju A. The prevalence of neuropsychiatric syndromes in systemic lupus erythematosus. Neurology. 2001;57(3):496-500.

16. Fiedorowicz-Fabrycy I, Brzosko M. Diagnostics of neuropsychiatric complications in vascular diseases in the course of systemic connective tissue diseases. Reumatologia. 2005;43:369-372.

17. Palagini L, Mosca M, Tani C, Gemignani A, Mauri M, Bombardieri S. Depression and systemic lupus erythematosus: A systematic review. Lupus. 2013;22(5):409-416. 